\title{
PSMA as a target for radiolabelled small molecules
}

\author{
Matthias Eder • Michael Eisenhut • John Babich • Uwe Haberkorn
}

Published online: 6 March 2013

(C) The Author(s) 2013. This article is published with open access at Springerlink.com

Prostate cancer (PCa) is the most common cancer in men in Europe. When detected early, and when the disease is localized to the prostate gland, the 5-year survival rate is nearly $100 \%$. However, once the cancer has spread beyond the prostate, survival rates fall dramatically [1]. In recent years there has been a significant decrease in mortality mainly due to early detection. However, this early detection has led to overdiagnosis and overtreatment with resultant impact on the quality of life of men with PCa [2]. These problems are due to the variability of the clinical course of the disease and the high prevalence of microscopic disease [3, 4]. Therefore, a risk-adapted strategy is needed to choose among a wide variety of treatment options, from active surveillance to aggressive treatment. In the face of such broadly differing options that impact survival and quality of life it follows that patient-specific staging is essential for optimizing individual outcomes. Patient-specific staging drives the demand for sensitive and specific imaging of $\mathrm{PCa}$ including intraprostatic disease as well as local and distant metastases. Furthermore, as active surveillance becomes a more widely considered management option in low-grade disease, a sensitive method for monitoring changes in tumour volume and location would potentially eliminate the need for repetitive biopsies and enable a more advanced temporal evaluation.

Evaluation of PSA kinetics is useful in assessing risk in those with first-line treatment failure, but is known to be

\author{
M. Eder · M. Eisenhut \\ Radiopharmaceutical Chemistry, German Cancer Research Center, \\ Im Neuenheimer Feld 280, \\ Heidelberg 69120, Germany \\ J. Babich \\ Molecular Insight Pharmaceuticals, Inc., 160 Second Street, \\ Cambridge, MA 02142, USA \\ U. Haberkorn $(\bowtie)$ \\ Department of Nuclear Medicine, University of Heidelberg, \\ Im Neuenheimer Feld 400, \\ Heidelberg 69120, Germany \\ e-mail: Uwe.Haberkorn@med.uni-heidelberg.de
}

unreliable for active surveillance [5]. A stable PSA level during the first 2 years after diagnosis does not preclude the possibility of lethal cancer [6-8]. Repeat biopsies which give information about tumour presence, size and grade may be performed to decide if and when therapy is needed, but it is uncertain what tumour size and grade thresholds should be used to instigate therapy. In other words, it is unclear how big a tumour or how high the Gleason score has to be to justify treatment. Furthermore, biopsy is associated with risks of bleeding and infection and patients undergoing multiple prostate biopsies are more likely to be diagnosed with clinically insignificant PCa than those who only undergo one biopsy [9].

Since molecular alterations in these tumours are responsible for their biological behaviour, efforts are underway to develop and validate markers that identify patients at risk. Genomics data may lead to a more accurate prediction of individual tumour behaviour, but still have not achieved widespread acceptance [4]. In addition these data are also based on biopsy results. In contrast, a noninvasive method of imaging PCa offers the possibility to perform repeated measurements of the volume and biological properties of a tumour which can be used for the specific staging of an individual man and guide the timing and selection of the most appropriate treatment. In essence, monitoring changes in disease burden anywhere it is found in the body may offer the best means of patient management.

There are several biological characteristics making prostate-specific membrane antigen (PSMA) an outstanding target for nuclear medicine. As a type II transmembrane protein with glutamate-carboxypeptidase activity and a known substrate, PSMA is an ideal target for developing small-molecule radiopharmaceuticals which typically show fast blood clearance and low background activity. Furthermore, upon ligand binding, PSMA is internalized via clathrin-coated pits and subsequent endocytosis [10] resulting in an effective transportation of the bound molecule into the cells. Since internalization leads to enhanced tumour uptake and retention, targeting PSMA is expected to 
result in high image quality. Finally, PSMA is a cell-surface protein that shows a significant over-expression on prostatic cancer cells and especially in advanced stage prostate carcinomas with low expression in normal human tissue [11, 12]. There are several corroborating studies showing that PSMA expression levels increase according to the stage and grade of the tumour [12-14]. Moreover, in nearly all adenocarcinomas of the prostate the majority of primary and metastatic lesions show PSMA expression [14, 15]. Interestingly, PSMA expression has also been reported in carcinomas of the colon and breast, and in those of renal origin in newly formed blood vessels $[12,16]$, which might affect the therapeutic potential of PSMA-targeting molecules. Taking these findings together, PSMA seems to be an ideal target for high-contrast nuclear (PET/CT and SPECT/CT) imaging, and therefore has high potential to improve patient management at every stage of the disease.

Currently, a radiolabelled anti-PSMA antibody (ProstaScint, capromab pendetide; EUSA Pharma, Langhorne, PA) is approved in the USA for the detection of soft-tissue metastasis and recurrence of PCa. However, this antibody targets the intracellular domain of PSMA, limiting its utility [17]. Clinical trial results with the antiPSMA monoclonal antibody J591 have shown improved targeting of $\mathrm{PCa}[18,19]$. Although antibodies offer potential for tumour targeting, their effectiveness as diagnostic radiopharmaceuticals is limited by a long circulating halflife and poor tumour penetrability, particularly for bone metastases. There are promising approaches that may overcome these limitations, such as combining antibodies with longer-lived PET radioisotopes such as ${ }^{89} \mathrm{Zr}$ and ${ }^{64} \mathrm{Cu}[20$, 21] or using smaller variants such as single chain fragments. Apart from diagnosis, however, antibodies directed against PSMA may have an adjuvant therapeutic impact as they are able to recruit cells of the immune system.

Early work on the development of inhibitors of the enzyme $\mathrm{N}$-acetylaspartylglutamate peptidase or NAAALDASE, a glutamate carboxypeptidase II enzyme, identified a number of small molecule inhibitors of this enzyme such as those described by Jackson et al. [22, 23]. Ultimately, the identification of the structural [24] and functional [25] homology between NAALADASE and PSMA opened the possibility of using these small molecules in the targeted treatment and imaging of PCa. The "active substrate recognition site" of PSMA is composed of two structural motifs, one recognizing the glutarate moiety of glutamic acid inherent in NAAG while the other, a more promiscuous site lined with basic amino acids, allows binding to the free carboxylate of aspartate and can accommodate more bulky structural moieties [26]. Molecules lacking one of these interactions have shown reduced binding and internalization rates [27]. As a consequence, the design of functionally active molecules is complex, especially when a bulky chelator has to be introduced to incorporate radiometals. Subsequent to these reports several groups have reported on the development of small-molecule inhibitors of PSMA labelled with ${ }^{123} \mathrm{I}$ [28-30], ${ }^{99 \mathrm{~m}} \mathrm{Tc}[31,32],{ }^{18} \mathrm{~F}$ [33], ${ }^{111} \mathrm{In}[34]$ and ${ }^{68} \mathrm{Ga}$ [35] based on the structural motifs of various NAALADASE inhibitors.

The first high-affinity small-molecule inhibitors of PSMA, ${ }^{123}$ I-MIP-1072 and ${ }^{123}$ I-MIP-1095, were introduced into the clinic in 2008. In men with metastatic PCa, SPECT/CT using these molecules demonstrated the ability to rapidly detect lesions in soft tissue, bone and the prostate gland as early as $1-4 \mathrm{~h}$ after injection [36]. ${ }^{123}$ I-MIP-1072 was subsequently evaluated in a chemotherapy model of $\mathrm{PCa}$ and clearly demonstrated that tumour uptake is directly proportional to viable tumour mass providing the potential to track changes in response to therapy. Hence this imaging approach may satisfy a critical unmet need in the evaluation of PCa [37]. The results of the initial clinical investigation also led to the evaluation of these iodine-containing ligands as potential PSMA-targeted radiotherapeutics when labelled with ${ }^{131} \mathrm{I}[38,39]$. The growth of spheroids of human PCa LNCaP cells and LNCaP tumour xenografts has been shown to be inhibited in a dose-dependent manner by treatment with ${ }^{131}$ I-labelled analogues of these compounds, suggesting the potential for a unique therapeutic approach with these small molecules. PET studies with ${ }^{124}$ I-MIP-1095 have shown high accumulation in tumour lesions, excellent contrast (Fig. 1a) and favourable dosimetry. The first therapeutic use in patients showed long-lasting tracer accumulation in tumour lesions (Fig. 1b) and a response with respect to PSA values and pain symptoms in most patients (data not shown).

However, the answer to the principle question as to whether there is a clinical demand for PSMA-directed imaging depends on how such imaging compares with substances and techniques already in clinical use. Since a large group of patients show biochemical relapse after radical prostatectomy, one major indication for imaging of $\mathrm{PCa}$ is the localization of recurrent disease. The early differentiation between local disease and distant metastasis is of high importance for patient management and the therapeutic procedure. At PSA levels higher than $2 \mathrm{ng} / \mathrm{mL}$, salvage radiation therapy is almost ineffective after prostatectomy. Thus, radiotracers able to image lesions at low PSA levels are of particular clinical interest. ${ }^{18} \mathrm{~F}$-Labelled choline is established in many clinical institutions as it is able to distinguish PCa lesions with moderate to good sensitivity in a large group of patients [40, 41]. However, the value of choline for the detection of recurrent $\mathrm{PCa}$ is limited in patients with PSA levels $<2.5 \mathrm{ng} / \mathrm{mL}$ [40, 41]. In patients with a PSA level less than $1 \mathrm{ng} / \mathrm{mL}$ the probability of positive PET findings using choline is only $19 \%$ as reported by Giovacchini et al. [40]. Similarly, ${ }^{11} \mathrm{C}$-acetate shows a 


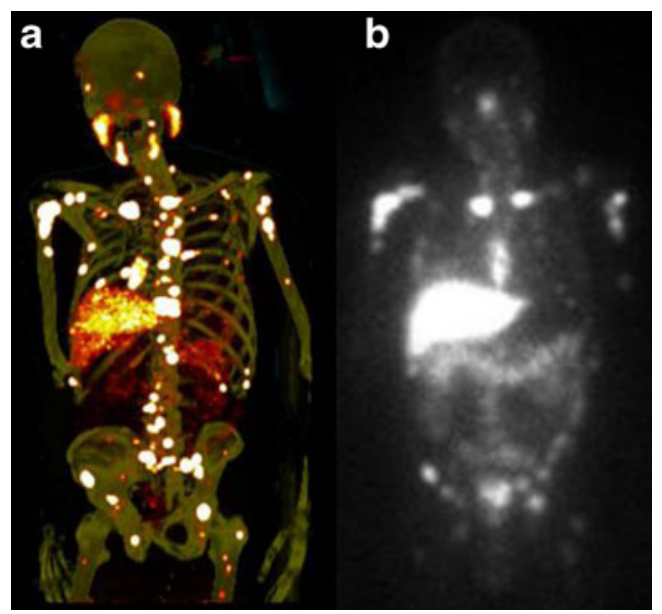

Fig. 1 a Maximum intensity projection image of a PET scan performed on day 5 after administration of ${ }^{124}$ I-MIP-1095 shows multiple lesions in bones and lymph nodes, and also accumulation in the salivary and lacrimal glands. b Whole-body scan 7 days after administration of $5 \mathrm{GBq}{ }^{131} \mathrm{I}$-MIP-1466 in the same patient

poor detection rate in patients with low PSA levels [42]. Considering that biochemical relapse is already expected in those with a PSA level of $0.2 \mathrm{ng} / \mathrm{mL}$, there is a high demand for more sensitive compounds.

The first clinical results using a ${ }^{68} \mathrm{Ga}$-labelled small molecule targeting PSMA [35, 43, 45] suggest a high potential for the detection of small recurrent PCa lesions in patients presenting with low PSA values. As expected from the biological characteristics including the expression pattern and the potential to internalize upon binding PCa cells, the ${ }^{68} \mathrm{Ga}$-labelled PSMA inhibitor GluNH-CO-NH-Lys(Ahx)-HBED-CC shows high accumulation in small metastases and is cleared rapidly from background tissue. In direct comparison to ${ }^{18} \mathrm{~F}$-labelled choline, our first results suggest that PSMA-targeted imaging is able to detect lesions much earlier in patients with low PSA values and shows a reduced background activity in healthy tissue (Fig. 2). Considering the growing accessibility of ${ }^{68} \mathrm{Ga}$ from a generator and the ease of labelling, ${ }^{68} \mathrm{Ga}$ PSMA-targeted imaging has high potential to become a valuable diagnostic agent in recurrent $\mathrm{PCa}$ and therapy monitoring. MIP-1404, a ${ }^{99 \mathrm{~m}}$ Tc-labelled small molecule inhibitor of PSMA also shows high potential for the detection of tumour lesions and offers even more widespread use in clinical practice (Fig. 3).

In the context of primary diagnosis the value of PSMAtargeted imaging has to be assessed in studies with histological backup because PSMA is expressed to a moderate level in normal prostate cells. Currently, ${ }^{99 \mathrm{~m}} \mathrm{Tc}-\mathrm{MIP}-1404$ is under evaluation in an international phase 2 study in men scheduled for radical prostatectomy at high risk of lymph node involvement. The study will evaluate the ability of
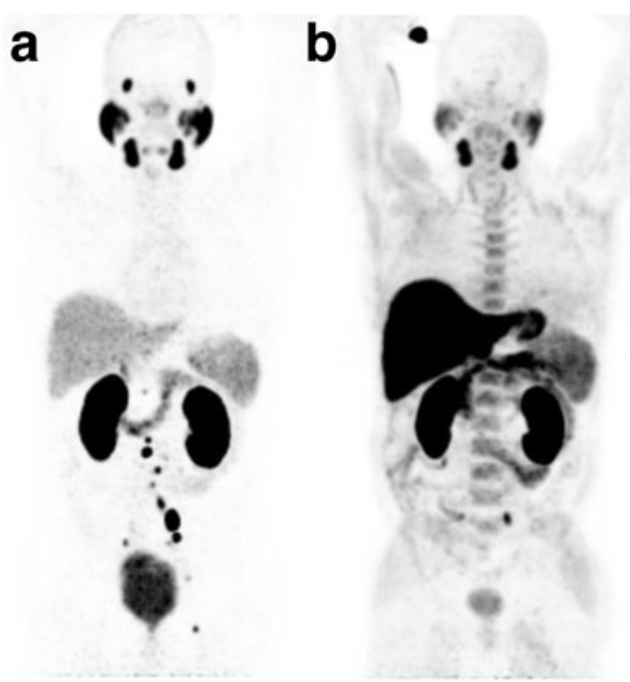

Fig. 2 PET images in the same patient using the ${ }^{68} \mathrm{Ga}$-labelled PSMA inhibitor Glu-NH-CO-NH-Lys(Ahx)-HBED-CC (a) and ${ }^{18} \mathrm{~F}-$ fluoroethylcholine (b). The scan with the PSMA ligand shows significantly more lesions than the fluoroethylcholine scan in which only one metastasis is seen

MIP-1404 to detect disease using histopathology as the gold standard (ClinicalTrials.gov Identifier NCT01667536). Furthermore, many promising efforts such as magnetic resonance tomography to improve primary diagnosis are ongoing. Further studies are required to determine if PSMAtargeted imaging is able to supplement these modalities.

Thus we can address the question as to the clinical need for PSMA-targeted imaging. In an editorial comment Scher and Seitz wrote in 2008: "The crux of the matter is that, regarding further therapeutic options, the patient group [with low PSA levels] would presumably benefit the most from early identification of the site of recurrence" [44]. Since choline PET/CT is not able to image PCa recurrence at these low PSA levels, a more sensitive compound based on PSMA-targeted imaging is needed to become the new clinical standard.

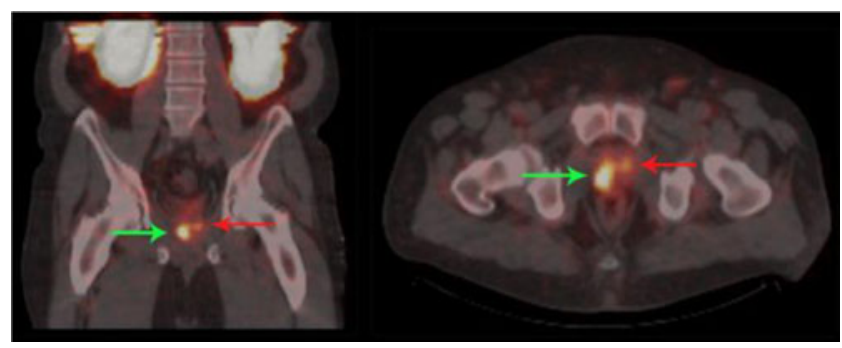

Fig. 3 SPECT/CT fusion images in a patient with biopsy-proven $\mathrm{PCa}$ and a PSA level of $8.5 \mathrm{ng} / \mathrm{mL}$. The images were acquired 2 weeks prior to surgery. The scan was performed $4 \mathrm{~h}$ after injection of $740 \mathrm{MBq}$ ${ }^{99 \mathrm{~m}} \mathrm{Tc}-\mathrm{MIP}-1404$. Bilateral disease is visualized and was confirmed on pathology with one lesion showing a Gleason score of 7 (green arrow) and the other a Gleason score of 6 (red arrow) 
Open Access This article is distributed under the terms of the Creative Commons Attribution License which permits any use, distribution, and reproduction in any medium, provided the original author(s) and the source are credited.

\section{References}

1. National Cancer Institute, Surveillance Epidemiology and End Results. SEER stat fact sheets: prostate. Available from: http:// seer.cancer.gov/statfacts/html/prost.html.

2. Resnick MJ, Koyama T, Fan KH, Albertsen PC, Goodman M, Hamilton AS, et al. Long-term functional outcomes after treatment for localized prostate cancer. N Engl J Med. 2013;368:436-45.

3. Pinto F, Totaro A, Palermo G, Calarco A, Sacco E, D'Addessi A, et al. Imaging in prostate cancer staging: present role and future perspectives. Urol Int. 2012;88:125-36.

4. Klotz L. Cancer overdiagnosis and overtreatment. Curr Opin Urol. 2012;22:203-9.

5. Ross AE, Loeb S, Landis P, Partin AW, Epstein JI, Kettermann A, et al. Prostate-specific antigen kinetics during follow-up are an unreliable trigger for intervention in a prostate cancer surveillance program. J Clin Oncol. 2010;28:2810-6.

6. Lees K, Durve M, Parker C. Active surveillance in prostate cancer: patient selection and triggers for intervention. Curr Opin Urol. 2012;22:210-5.

7. Loblaw A, Zhang L, Lam A, Nam R, Mamedov A, Vesprini D, et al. Comparing prostate specific antigen triggers for intervention in men with stable prostate cancer on active surveillance. J Urol. 2010;184:1942-6.

8. Fall K, Garmo H, Andren O, Bill-Axelson A, Adolfsson J, Adami HO, et al. Prostate-specific antigen levels as a predictor of lethal prostate cancer. J Natl Cancer Inst. 2007;99:526-32.

9. Park BK, Park JW, Park SY, Kim CK, Lee HM, Jeon SS, et al. Prospective evaluation of 3-T MRI performed before initial transrectal ultrasound-guided prostate biopsy in patients with high prostate-specific antigen and no previous biopsy. AJR Am J Roentgenol. 2011;197:W876-81.

10. Rajasekaran SA, Anilkumar G, Oshima E, Bowie JU, Liu H, Heston W, et al. A novel cytoplasmic tail MXXXL motif mediates the internalization of prostate-specific membrane antigen. Mol Biol Cell. 2003;14:4835-45.

11. Perner S, Hofer MD, Kim R, Shah RB, Li H, Moller P, et al. Prostate-specific membrane antigen expression as a predictor of prostate cancer progression. Hum Pathol. 2007;38:696-701.

12. Silver DA, Pellicer I, Fair WR, Heston WD, Cordon-Cardo C. Prostate-specific membrane antigen expression in normal and malignant human tissues. Clin Cancer Res. 1997;3:81-5.

13. Chang SS. Overview of prostate-specific membrane antigen. Rev Urol. 2004;6 Suppl 10:S13-8.

14. Bostwick DG, Pacelli A, Blute M, Roche P, Murphy GP. Prostate specific membrane antigen expression in prostatic intraepithelial neoplasia and adenocarcinoma: a study of 184 cases. Cancer. 1998;82:2256-61.

15. Mannweiler S, Amersdorfer P, Trajanoski S, Terrett JA, King D, Mehes G. Heterogeneity of prostate-specific membrane antigen (PSMA) expression in prostate carcinoma with distant metastasis. Pathol Oncol Res. 2009;15:167-72.

16. Chang SS, O'Keefe DS, Bacich DJ, Reuter VE, Heston WD, Gaudin PB. Prostate-specific membrane antigen is produced in tumorassociated neovasculature. Clin Cancer Res. 1999;5:2674-81.

17. Troyer JK, Beckett ML, Wright Jr GL. Location of prostatespecific membrane antigen in the LNCaP prostate carcinoma cell line. Prostate. 1997;30:232-42.
18. Milowsky MI, Nanus DM, Kostakoglu L, Sheehan CE, Vallabhajosula S, Goldsmith SJ, et al. Vascular targeted therapy with anti-prostate-specific membrane antigen monoclonal antibody J591 in advanced solid tumors. J Clin Oncol. 2007;25:540-7.

19. Vallabhajosula S, Goldsmith SJ, Kostakoglu L, Milowsky MI, Nanus DM, Bander NH. Radioimmunotherapy of prostate cancer using 90Y- and 177Lu-labeled J591 monoclonal antibodies: effect of multiple treatments on myelotoxicity. Clin Cancer Res. 2005; $11: 7195$ s-200s.

20. Holland JP, Divilov V, Bander NH, Smith-Jones PM, Larson SM, Lewis JS. 89Zr-DFO-J591 for immunoPET of prostate-specific membrane antigen expression in vivo. J Nucl Med. 2010;51:1293-300.

21. Elsasser-Beile U, Reischl G, Wiehr S, Buhler P, Wolf P, Alt K, et al. PET imaging of prostate cancer xenografts with a highly specific antibody against the prostate-specific membrane antigen. $\mathrm{J}$ Nucl Med. 2009;50:606-11.

22. Jackson PF, Cole DC, Slusher BS, Stetz SL, Ross LE, Donzanti BA, et al. Design, synthesis, and biological activity of a potent inhibitor of the neuropeptidase $\mathrm{N}$-acetylated alpha-linked acidic dipeptidase. J Med Chem. 1996;39:619-22.

23. Jackson PF, Slusher BS. Design of NAALADase inhibitors: a novel neuroprotective strategy. Curr Med Chem. 2001;8:94957.

24. Luthi-Carter R, Barczak AK, Speno H, Coyle JT. Molecular characterization of human brain N-acetylated alpha-linked acidic dipeptidase (NAALADase). J Pharmacol Exp Ther. 1998;286:1020-5.

25. Tiffany CW, Lapidus RG, Merion A, Calvin DC, Slusher BS. Characterization of the enzymatic activity of PSM: comparison with brain NAALADase. Prostate. 1999;39:28-35.

26. Mesters JR, Barinka C, Li W, Tsukamoto T, Majer P, Slusher BS, et al. Structure of glutamate carboxypeptidase II, a drug target in neuronal damage and prostate cancer. EMBO J. 2006;25:1375-84.

27. Liu T, Toriyabe Y, Kazak M, Berkman CE. Pseudoirreversible inhibition of prostate-specific membrane antigen by phosphoramidate peptidomimetics. Biochemistry. 2008;47:12658-60.

28. Maresca KP, Hillier SM, Femia FJ, Keith D, Barone C, Joyal JL, et al. A series of halogenated heterodimeric inhibitors of prostate specific membrane antigen (PSMA) as radiolabeled probes for targeting prostate cancer. J Med Chem. 2009;52:347-57.

29. Hillier SM, Maresca KP, Femia FJ, Marquis JC, Foss CA, Nguyen N, et al. Preclinical evaluation of novel glutamate-urea-lysine analogues that target prostate-specific membrane antigen as molecular imaging pharmaceuticals for prostate cancer. Cancer Res. 2009;69:6932-40.

30. Foss CA, Mease RC, Fan H, Wang Y, Ravert HT, Dannals RF, et al. Radiolabeled small-molecule ligands for prostate-specific membrane antigen: in vivo imaging in experimental models of prostate cancer. Clin Cancer Res. 2005;11:4022-8.

31. Lu G, Maresca KP, Hillier SM, Zimmerman CN, Eckelman WC, Joyal JL, et al. Synthesis and SAR of $(99 \mathrm{~m}) \mathrm{Tc} /$ Re-labeled small molecule prostate specific membrane antigen inhibitors with novel polar chelates. Bioorg Med Chem Lett. 2013;23:1557-63.

32. Kularatne SA, Zhou Z, Yang J, Post CB, Low PS. Design, synthesis, and preclinical evaluation of prostate-specific membrane antigen targeted $(99 \mathrm{~m}) \mathrm{Tc}$-radioimaging agents. Mol Pharm. 2009;6:790-800.

33. Chen Y, Pullambhatla M, Byun Y, Foss CA, Nimmagadda S, Senthamizhchelvan S, et al. 2-(3-\{1-Carboxy-5-[(6-[18F]fluoropyridine-3-carbonyl)-amino]-pentyl $\}$-ureido)-pentanedioic acid, [18F]DCFPyL, a PSMA-based PET imaging agent for prostate cancer. Clin Cancer Res. 2011;17:7645-53.

34. Banerjee SR, Pullambhatla M, Shallal H, Lisok A, Mease RC, Pomper MG. A modular strategy to prepare multivalent inhibitors of prostate-specific membrane antigen (PSMA). Oncotarget. 2011;2:1244-53. 
35. Afshar-Oromieh A, Haberkorn U, Eder M, Eisenhut M, Zechmann C. [(68)Ga]Gallium-labelled PSMA ligand as superior PET tracer for the diagnosis of prostate cancer: comparison with (18)F-FECH. Eur J Nucl Med Mol Imaging. 2012;39:1085-6.

36. Barrett JA, Coleman RE, Goldsmith SJ, Vallabhajosula S, Petry NA, Cho S, et al. First-in-man evaluation of two high-affinity PSMA-avid small molecules for imaging prostate cancer. J Nucl Med. 2013. doi:10.2967/jnumed.112.111203

37. Hillier SM, Kern AM, Maresca KP, Marquis JC, Eckelman WC, Joyal JL, et al. 123I-MIP-1072, a small-molecule inhibitor of prostate-specific membrane antigen, is effective at monitoring tumor response to taxane therapy. J Nucl Med. 2011;52:1087-93.

38. Hillier S, Rubino K, Maresca K, Marquis J, Tesson M, Zimmerman C, et al. [131I]MIP-1466, a small molecule prostate-specific membrane antigen (PSMA) inhibitor for targeted radiotherapy of prostate cancer (PCa). J Nucl Med. 2012;53 Suppl $1: 170$.

39. Hillier S, Merkin R, Maresca K, Zimmerman C, Barrett J, Tesson M, et al. [131I]MIP-1375, a small molecule prostate-specific membrane antigen (PSMA) inhibitor for targeted therapy of prostate cancer (PCa). J Nucl Med. 2011;52 Suppl 1:361.
40. Giovacchini G, Picchio M, Coradeschi E, Bettinardi V, Gianolli L, Scattoni V, et al. Predictive factors of $[(11) C]$ choline PET/CT in patients with biochemical failure after radical prostatectomy. Eur J Nucl Med Mol Imaging. 2010;37:301-9.

41. Rinnab L, Simon J, Hautmann RE, Cronauer MV, Hohl K, Buck $\mathrm{AK}$, et al. [(11)C]choline PET/CT in prostate cancer patients with biochemical recurrence after radical prostatectomy. World J Urol. 2009;27:619-25.

42. Albrecht S, Buchegger F, Soloviev D, Zaidi H, Vees H, Khan HG, et al. (11)C-acetate PET in the early evaluation of prostate cancer recurrence. Eur J Nucl Med Mol Imaging. 2007;34:185-96.

43. Afshar-Oromieh A, Malcher A, Eder M, Eisenhut M, Linhart HG, Hadaschik BA, et al. PET imaging with a [(68)Ga]gallium-labelled PSMA ligand for the diagnosis of prostate cancer: biodistribution in humans and first evaluation of tumour lesions. Eur J Nucl Med Mol Imaging. 2012. doi:10.1007/s00259-012-2298-2.

44. Scher B, Seitz M. PET/CT imaging of recurrent prostate cancer. Eur J Nucl Med Mol Imaging. 2008;35:5-8.

45. Eder M, Schafer M, Bauder-Wust U, Hull WE, Wangler C, Mier W, et al. (68)Ga-Complex Lipophilicity and the Targeting Property of a Urea-Based PSMA Inhibitor for PET Imaging. Bioconjug Chem. 2012;23:688-97. 